\section{Risk Factors for Cardiovascular Disease in Rheumatoid Arthritis: Defining Treatment Thresholds}

\section{To the Editor:}

We read with interest the very insightful editorial by Dessein, et al ${ }^{1}$ on the determinants of dyslipidemia in rheumatoid arthritis (RA) in a recent issue of The Journal. There is little doubt that dyslipidemia participates in the atherogenesis of RA even when the disease is treated with disease-modifying antirheumatic drugs (DMARD), and its determinants comprise more of the conventional dyslipidemia risk factors than RA characteristics ${ }^{1}$. The question is how to best define the thresholds for conventional risk factors where interventions would be necessary.

Although cardiovascular disease (CVD) is highly prevalent in RA, identification is often difficult on clinical grounds alone, especially where reduced physical exertion and symptoms of CVD may be attributed to musculoskeletal rather than cardiac causes ${ }^{2}$. Further, it may be clinically silent in many patients, emphasizing the need for a high degree of awareness ${ }^{3}$. Close attention to the management of traditional cardiovascular risk factors is therefore essential in this group of patients ${ }^{4,5}$. Both rheumatologists and community physicians are therefore interested in knowing thresholds where treatment would be indicated for primary prevention of CVD in RA.

The modified Joint British Societies (JBS2) CVD risk calculator ${ }^{6}$ is a tool for estimating CVD risk (nonfatal myocardial infarction and stroke, coronary and stroke death, and new angina pectoris) for Caucasian individuals who have not already developed coronary heart disease or other major atherosclerotic disease based on age, sex, smoking habit, systolic blood pressure, and the ratio of total cholesterol to high-density lipoprotein (HDL) cholesterol. This tool is an aid to making clinical decisions about how intensively to intervene on lifestyle and whether to use antihypertensive, lipid-lowering, and antiplatelet medication. A total CVD risk of > $20 \%$ over 10 years is defined as high risk and requires professional lifestyle intervention and, where appropriate, drug therapies to achieve the lifestyle and risk factor targets. It is recommended that such calculated risk be doubled $^{7}$ to account for the excess risk associated with chronic inflammation in RA. We would like to share our experience in using this tool to assess the CVD risk in patients with RA.

Caucasian patients with RA were prospectively studied. All were assessed for age, sex, RA disease duration and activity and therapy, smoking status, body mass index, systolic blood pressure, fasting blood glucose, total and HDL cholesterol, family history of premature coronary heart disease or stroke, and preexisting cardiovascular comorbidity. Absolute 10 -year CVD risk was calculated using the JBS2 CVD risk calculator ${ }^{6}$, which is used for nondiabetic populations in all age groups.

Sixty-five patients [ 46 women ( $71 \%$ ); mean age $63 \pm 11.95$ yrs, mean disease duration $13.6 \pm 9.75 \mathrm{yrs}]$ were included. Thirty-seven $(57 \%)$ patients had known cardiovascular comorbidities (hypertension 29, angina 7 , myocardial infarction 3 , cerebrovascular accident 2 , peripheral vascular disease 3 , hyperlipidemia 18 , and diabetes 3 ). Table 1 gives details of individual risk factors in 28 (43\%) patients who had no known cardiovascular comorbidity. Absolute 10-year CVD risk is shown in Table 2 for this group. Fourteen percent (4/28) of RA patients were thus suitable for primary prevention interventions. However, that figure rose to $36 \%$ (10/28) when the calculated risk was doubled ${ }^{7}$ to account for the excess risk associated with chronic inflammation in RA (Table 2).

The concept of total CVD risk replaces the traditional dichotomous classification of risk factors in most people ${ }^{6}$. Inappropriate treatment of single risk factor in those at low total CVD risk is thus avoided. It is consonant with clinical practice in which physicians deal with the whole person rather than just one aspect of cardiovascular risk ${ }^{6}$. A person's CVD risk estimation can be further improved by taking account of other CVD risk factors not included in the calculator such as abdominal obesity, those with family history of premature CVD, and different ethnicity.

The JBS2 CVD risk calculator appears to be a useful tool to assess overall CVD risk in patients with RA and identifies those in whom primary prevention interventions are warranted.
Table 1. Twenty-eight patients with rheumatoid arthritis without known cardiovascular comorbidity: demographic and disease characteristics and individual risk factors.

\begin{tabular}{lc}
\hline Characteristics & Values \\
\hline Age, yrs (mean $\pm \mathrm{SD})$ & $58.82 \pm 13.11$ \\
Female, $\mathrm{n}(\%)$ & $22(79)$ \\
Disease duration, yrs (mean $\pm \mathrm{SD})$ & $10.67 \pm 7.21$ \\
DAS28, mean $\pm \mathrm{SD}$ & $2.5 \pm 0.7$ \\
TC/HDL cholesterol ratio, mmol/l (mean $\pm \mathrm{SD})$ & $3.73 \pm 0.83$ \\
Fasting glucose, $\mathrm{mmol} / \mathrm{l}($ mean $\pm \mathrm{SD})$ & $4.69 \pm 0.55$ \\
Body mass index, $\mathrm{kg} / \mathrm{m}^{2}($ mean $\pm \mathrm{SD})$ & $27.18 \pm 5.11$ \\
Systolic blood pressure, mm Hg (mean $\pm \mathrm{SD})$ & $128.67 \pm 13.59$ \\
Smokers, $\mathrm{n}(\%)$ & $9(32)$ \\
\hline
\end{tabular}

DAS: Disease Activity Score; TC: total cholesterol; HDL: high-density lipoprotein.

Table 2. Absolute 10-year cardiovascular risk in RA patients without known cardiovascular comorbidity. Includes 3 patients with family history of premature CVD, which increases the risk by a factor of 1.3 .

\begin{tabular}{lcc}
\hline CVD Risk & $\begin{array}{c}\text { RA Patients, } \\
\mathrm{n}=28\end{array}$ & $\begin{array}{c}\text { RA Patients,* } \\
\mathrm{n}=28\end{array}$ \\
\hline$<10 \%, \mathrm{n}(\%)$ & $15(54)$ & $8(28)$ \\
$10-20 \%, \mathrm{n}(\%)$ & $9(32)$ & $10(36)$ \\
$20-30 \%, \mathrm{n}(\%)$ & $4(14)$ & $7(25)$ \\
$>30 \%, \mathrm{n}(\%)$ & 0 & $3(11)$ \\
\hline
\end{tabular}

RA: rheumatoid arthritis; CVD: cardiovascular disease. * Number of RA patients after CVD risk was multiplied by 2 .

VINOD RAVINDRAN, MD, MRCP; RODNEY A. HUGHES, MD, FRCP, Department of Rheumatology, St. Peter's Hospital, Guildford Road, Chertsey, KT16 0PZ, United Kingdom. Address correspondence to Dr. V. Ravindran; E-mail: drvinod12@gmail.com

\section{REFERENCES}

1. Dessein PH, Christian BF, Solomon A. Which are the determinants of dyslipidemia in rheumatoid arthritis and does socioeconomic status matter in this context? [editorial]. J Rheumatol 2009;36:1357-61

2. Goodson N. Coronary artery disease and rheumatoid arthritis. Curr Opin Rheumatol 2002;14:115-20.

3. Banks MJ, Kitas GD. Patients' physical disability in RA may influence doctors' perceptions of suitability for risk assessment of CHD. BMJ 1999;319:1266.

4. Erb N, Pace AV, Douglas KMJ, Banks MJ, Kitas GD. Risk assessment for coronary heart disease in rheumatoid arthritis and osteoarthritis. Scand J Rheumatol 2004;33:293-9.

5. British Society for Rheumatology. BSR guidelines on standards of care for persons with rheumatoid arthritis. Rheumatology 2005;44:553-6.

6. Joint British Societies 2. Joint British Societies' guidelines on prevention of cardiovascular disease in clinical practice. Heart 2005;91 Suppl:1-52.

7. Symmons D, Bruce I. Management of cardiovascular risk in RA and SLE. Rheumatic disease hands on; (series 5) Number 8. Arthritis Research Campaign, UK 2006. [Internet. Accessed September 28, 2009.] Available at: www.arc.org.uk

J Rheumatol 2010;37:1; doi:10.3899/jrheum.090758 